\title{
Application of the Liquid-Crystal-Based Tunable Lyot Filter to the Optical Emission Imaging Plasma Spectrometry
}

\author{
Shinichiro KADO, Hiroshi SUZUKI ${ }^{1)}$, Yousuke KUWAHARA ${ }^{1)}$, Kiminori KURIHARA ${ }^{1)}$, \\ Taiichi SHIKAMA ${ }^{1)}$, Filippo SCOTTI ${ }^{1)}$, Yohei IIDA ${ }^{1)}$ and Satoru TANAKA ${ }^{1)}$ \\ High Temperature Plasma Center, The University of Tokyo, Kashiwa, 277-8568, Japan \\ ${ }^{1)}$ School of Engineering, The University of Tokyo, Tokyo 113-8656, Japan
}

(Received 9 December 2006 / Accepted 8 May 2007)

\begin{abstract}
An imaging spectrometry of plasmas using a tunable Lyot filter for the visible range $(400-720 \mathrm{~nm})$ is proposed. The typical passband and transmittance of the filter in the present study are $7 \mathrm{~nm}$ FWHM and $12 \%$ at $550 \mathrm{~nm}$. An optical system has been developed for a MAP-II divertor/edge plasma simulator. The line contamination was defined as the contributions of the adjacent lines in the passband of the filter and of the lines in the leak-band which were observed at longer than $548 \mathrm{~nm}$ at a specific wavelength setting below $455 \mathrm{~nm}$. We found that it is desirable to use a low-pass or band-pass filter to cut leak-bands, and that the He I lines of 447, 471, 492, 501 and $587 \mathrm{~nm}$ can be used for pure He plasma. Based on the CR model, the parameter range where these line ratios were applicable in our discharge condition of $12 \mathrm{mTorr}$ was evaluated for the electron temperature of $T_{\mathrm{e}}<20 \mathrm{eV}$ and the electron density of $10^{11}<n_{\mathrm{e}}<10^{14} \mathrm{~cm}^{-3}$.
\end{abstract}

(c) 2007 The Japan Society of Plasma Science and Nuclear Fusion Research

Keywords: Lyot filter, tunable wavelength filter, imaging spectrometry, fusion plasma, collisional radiative model, liquid-crystal filter, divertor simulator, MAP-II

DOI: $10.1585 /$ prr.2.S1125

\section{Introduction}

Line emissions from atoms, molecules and their ions in plasmas are useful to determine various properties of plasmas. The intensity ratio of different transitions offers, based on a collisional-radiative(CR) model [1], the electron temperature and density. In this case, a low-resolution wide-wavelength-range spectrometer $(\sim 0.1-1 \mathrm{~nm})$ is desirable. However, spectrometry using a grating monochromator in nature has a potential drawback in that the observable position is limited - a single point for a photo-multiplier tube (PMT) system or at most one-dimensional along the entrance slit for a charge-coupled device(CCD) system. Since the plasma parameters can dynamically change their spatial profile both along and orthogonally to the magnetic and/or flow fields, the necessity for an imaging system has been made apparent. Conventional imaging spectrometry employing the interference filter provides a single transmission wavelength for each filter. In order to increase the number of lines to be analyzed, however, one has to design so as to mechanically switch the filter or increase the viewing chord. The former requires a complicate system while the latter not only costs a great deal but these observing positions cannot be exactly identical. Nevertheless, the number of lines are still limited.

In this paper, we propose an imaging spectrometry using a wavelength-tunable liquid-crystal filter combined with the CR-model for He I emission for the application of the diagnostics of the divertor/edge region in fusion-

author'se-mail: kado@q.t.u-tokyo.ac.jp) relevant plasmas.

\section{Imaging System with a Lyot Filter}

The Lyot filter, originally proposed by Lyot [2] in 1933, is a kind of birefringent filter that has recently been widely employed in spectrometry for solar imaging [3], remote sensing [4], biochemistry, surface analysis, laser microscopy, etc. The technology of this filter, a recent review of which can be found in ref. [5] and the references therein, is based on polarization interference, the transmittance of which depends on the wavelength. The Lyot filter consists of several units. Each unit is assembled by a quartz fixed retarder and a nematic liquid crystal variable retarder, both of which are sandwiched together between a pair of linear polarizers having the same polarization direction. The optic axis of these retarders is 45 degree with respect to the polarizaion direction of the polarizers, which enables the maximum suppression capability against the light having the polarization orthogonal to that of the second polarizer at the exit of the unit. The retardation depends on the reciprocal of the wavelength, but in addition, it can be controlled by the applying bias to the nematic liquid crystal. The retardation of the fixed retarder is designed so that the total retardation for the target wavelength is an integer times $\pi$. - increasing the order narrows the free spectrum range - . By cascading this unit by varying the retardation order (the output light should be linearly polarized), the side-bands of higher interference are reduced. The trans- 
mission wavelength is tunable by varying the retardation of the nematic liquid crystals in each unit.

We are intending to apply this filter (Varispec: VIS-720), $7 \mathrm{~nm}$ in a typical passband at $550 \mathrm{~nm}$ and $20 \mathrm{~mm}$ in diameter, to the imaging spectrometry for the plasma optical emission. The optical system in the present study is shown in Fig. 1. Emission from the plasma at each wavelength passing through the filter is imaged onto the cooled CCD detector (Roper: EEV $1024 \times 256$ pixels) using a camera lens (Nikon) $50 \mathrm{~mm}$ in focal length and 1.2 in F-number. The size of each pixel of the CCD is $26 \mu \mathrm{m}$.

Magnification of the optical system is 0.084 , yielding an observation area of $320 \times 80 \mathrm{~mm}^{2}$. Since the view is limited by the diameter of the filter, however, the area is limited to about $210 \times 80 \mathrm{~mm}^{2}$.

The transmission function of the filter was determined by comparing the spectra of the light source (tungsten filament lamp) measured with and without inserting the filter using a low-resolution monochromator $(\Delta \lambda \sim 0.5 \mathrm{~nm})$, and was revealed to be described well by a Gaussian function. The passband in full width at half maximum (FWHM) and the transmittance at the peak wavelength are shown in Fig. 2. In addition, at certain wavelength setting below $455 \mathrm{~nm}$, a photon at the different wavelength was transmitted. This "leak-band" in the Lyot filter appears longer than $548 \mathrm{~nm}$. Although the transmittance of the leak-band

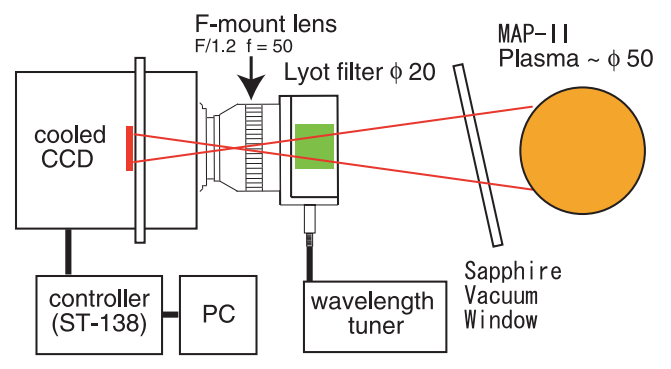

Fig. 1 Schematic view of the imaging spectrometry optics using Lyot filter.

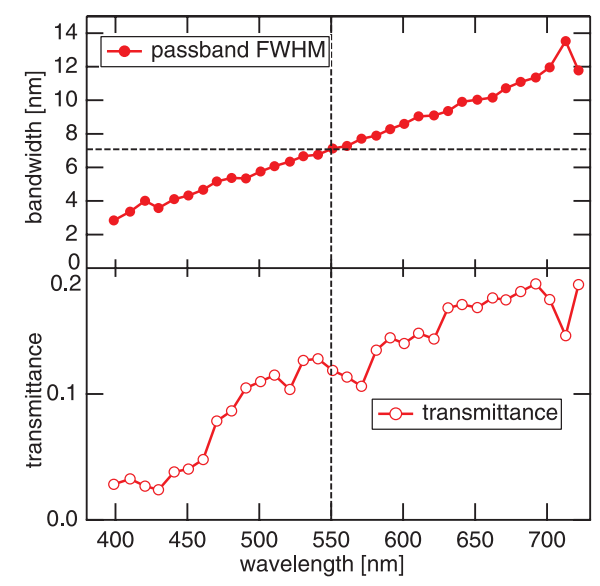

Fig. 2 Passband and transmittance of the Lyot filter. is small (typically less than a few percent of that of the main band), one should be careful in such cases when calibrating the intensity, when the line emission at the leak band has strong intensity, and when the quantum efficiency of the detector is high at the wavelength of the leak-band.

\section{Evaluation of the Line Contamina- tion and the Selection of the Line for He I}

After the relative intensity calibration, the intensity ratio between the images at different wavelengths can be obtained for each pixel of the CCD, which enables the application of the CR model [6] to determine the electron density or temperature distribution in the entire the observation area.

A concern of the filter system is, however, the superposition of the different line spectra, since there is no way to detect the spectral contaminants, which would be possible by the line profile in the case of monochromator systems. Therefore, we evaluated the usable lines of the He I emission from the view point of the lines that were free from the blend of other adjacent lines. The Gaussian transmission function of the filter, at the wavelength of the target line, is described as

$$
f\left(\lambda_{0} ; \lambda\right)=\exp \left(-\left(\frac{\lambda-\lambda_{0}}{\Delta w_{\text {filter }}}\right)^{2}\right),
$$

which is deconvoluted from that measured with the monochromator. The schematic procedure to determine the "line contamination", denoted as $\xi$, is shown in Fig. 3. The $i$-th spectral contaminant labeled $i(\geq 1)$ to the target line $(i=0)$ were evaluated as the intensity, namely $I_{i}=\sqrt{\pi} A_{i} \Delta w_{i}$, where $A_{i}$ and $\Delta w_{i}$ denote the amplitude and the broadening (half width at 1/e: hw1e) of the line, respectively. It should be mentioned that the lines at the leak-band can also contaminate the line to be measured.

The line contamination, or the line purity, defined by $1 /(1+\xi)$, can be evaluated both theoretically based on the CR model and experimentally from the spectra measured using a monochromator. The former gives the potentiality of the use of the line while the latter is the measure of

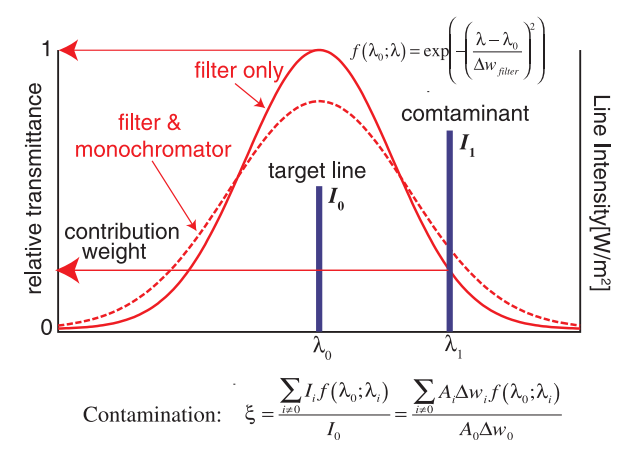

Fig. 3 Definition of the line contamination. 
the practical usage since other impurity lines can be superposed. After the relative intensity calibration both for the Lyot filter system and the monochromator using a standard lamp, one can confirm whether both results give the same intensity ratio, i.e., whether the intensity measured by the Lyot filter imaging system at the target wavelength divided by the target line intensity measured by the monochromator is constant for all line emissions or not. The result of the experimental evaluation using the He discharge lamp is shown in Fig. 4.

The lines evaluated based on the CR-model calculation and those based on the experiment are listed in Table 1 together with the information about the leak-band. $\xi_{C R}$ (main, leak) are the contamination in the main and leak bands, respectively. "CR" represents the CR-model calculation using the typical parameters in the MAP-II (the he-

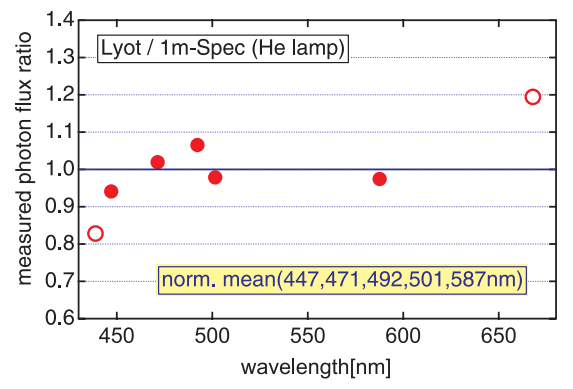

Fig. 4 Experimental line contamination represented as the ratio of the photon flux measured by the Lyot filter system to that by the grating monochromator for the He discharge lamp. Values are normalized to the average of the determined usable lines (filled circles) for visual purposes. The calibration factor for the 438 and $447 \mathrm{~nm}$ lines may suffer the effect of the leak band to some extent.

Table 1 Evaluation of the He I lines usable for the Lyot filter system. "ok" and "x" denote the "usable" and "useless", respectively, while "na" denotes "not available" due to experimental restrictions. "ok*" means that the line can be usable by using a low-pass filter to cut the leak bands.

\begin{tabular}{|l|l|l|l|l|l|l|}
\hline line[nm] & term & $\begin{array}{l}\mathrm{FW} \\
\mathrm{HM}\end{array}$ & $\begin{array}{l}\xi_{\mathrm{CR}} \\
\text { (main) }\end{array}$ & $\begin{array}{l}\xi_{\mathrm{CR}} \\
(\text { leak })\end{array}$ & CR & Exp \\
\hline 438.793 & $5^{1} \mathrm{D}$ & 4.0 & 0.011 & $5.9 \mathrm{E}-09$ & ok & $\begin{array}{l}\mathrm{x} \\
\mathrm{ok}\end{array}$ \\
\hline 443.755 & $5^{1} \mathrm{~S}$ & 4.2 & 1.8 & 0.0 & $\mathrm{x}$ & - \\
\hline 447.147 & $4^{3} \mathrm{D}$ & 4.2 & 0.014 & 0.039 & ok & ok \\
\hline 471.314 & $4^{3} \mathrm{~S}$ & 5.0 & 0.0 & - & ok & ok \\
\hline 492.193 & $4^{1} \mathrm{D}$ & 5.5 & $7.7 \mathrm{E}-4$ & - & ok & ok \\
\hline 501.568 & $3^{1} \mathrm{P}$ & 5.8 & 0.082 & - & ok & ok \\
\hline 504.774 & $4^{1} \mathrm{~S}$ & 6.0 & 2.40 & - & $\mathrm{x}$ & - \\
\hline 587.562 & $3^{3} \mathrm{D}$ & 8.3 & 0.0 & - & ok & ok \\
\hline 667.815 & $3^{1} \mathrm{D}$ & 10.7 & 0.0 & - & ok & $\mathrm{x}$ \\
\hline 706.518 & $3^{3} \mathrm{~S}$ & 11.8 & 0.0 & - & ok & na \\
\hline
\end{tabular}

lium pressure, $p_{\mathrm{He}}=12 \mathrm{mTorr}$, the gas temperature, $T_{\mathrm{gas}}=$ $400 \mathrm{~K}$, the effective absorption length of the radiation trapping, $L=25 \mathrm{~mm}$, the electron temperature $T_{\mathrm{e}}=3 \mathrm{eV}$, and the electron density $n_{\mathrm{e}}=10^{12} \mathrm{~cm}^{-3}$ ). "EXP" denotes the experimental evaluations using a helium discharge lamp - say, within the error of $10 \% \cdot 2^{3} \mathrm{P}-4^{3} \mathrm{D}(447 \mathrm{~nm})$ is useful since it has strong intensity compared with other lines from the principal quantum number $n=4$. Although the absolute photon flux in the present experiment cannot be determined due to the inaccuracy of the calibration factor with the leak-band, this line is potentially usable by inserting the low-pass filter to get rid of the leak-band. Popular lines such as $2^{1} \mathrm{P}-4{ }^{1} \mathrm{~S}(504 \mathrm{~nm})[7,8]$ cannot be used since the adjacent line of $501 \mathrm{~nm}$ has much stronger intensity: in contrast, the effect of the $504 \mathrm{~nm}$ line on the $501 \mathrm{~nm}$ line setting is negligible. The $2{ }^{1} \mathrm{P}-3{ }^{1} \mathrm{~S}$ line $(728 \mathrm{~nm})[7,9]$ also cannot be used because the visible Lyot filter cannot transmit this wavelength (out of controllable range). Therefore, we recommend using a low-pass filter $(<600 \mathrm{~nm})$ and applying $2^{3} \mathrm{P}-4^{3} \mathrm{D}(447 \mathrm{~nm}), 2^{3} \mathrm{P}-4^{3} \mathrm{~S}(471 \mathrm{~nm}), 2^{1} \mathrm{P}-$ $4^{1} \mathrm{D}(492 \mathrm{~nm}), 2{ }^{1} \mathrm{~S}-3^{1} \mathrm{P}(501 \mathrm{~nm})$, and $2^{3} \mathrm{P}-3^{3} \mathrm{D}(587 \mathrm{~nm})$.

\section{Applicability of the Lines Ratios}

In Fig. 5, we investigate the applicability of the line intensity ratios between the selected lines based on the CR model for He I. In the calculation including the radiation trapping using Otsuka's formula [10], we assume $p_{\mathrm{He}}=12 \mathrm{mTorr}, T_{\text {gas }}=400 \mathrm{~K}$ (rotational temperature of $\mathrm{H}_{2}$ for the Fulcher band spectroscopy [11]), and $L=$ $25 \mathrm{~mm}$ (core plasma radius) [12]. The contours in the figure represent the same population ratio as a function of $T_{\mathrm{e}}$ and $n_{\mathrm{e}}$. The regions where the contours are parallel to the vertical and horizontal axes represent the high sensitivity
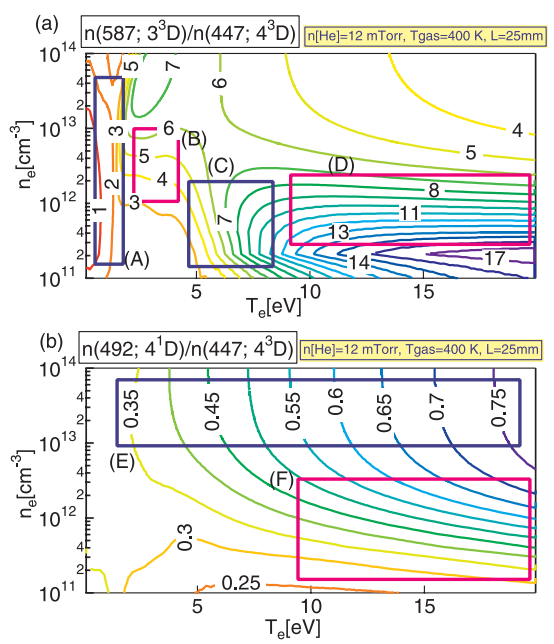

Fig. 5 Evaluation of the sensitivity of the population ratio to the electron density and temperature for the measurement using the line intensity ratio. The squares indicate the usable regions for the parameter measurement by the line intensity ratio method. 
only to $T_{\mathrm{e}}$ and $n_{\mathrm{e}}$, respectively, meaning the ratio is suitable for the measurement of $T_{\mathrm{e}}$ and $n_{\mathrm{e}}$, respectively. When there is no such region with parallel contours, the best-fit method can be applied to determine the parameters. This procedure, however, requires a much longer time to calculate, and is thus not suitable for the imaging spectrometry. Considering the application to the divertor/edge plasmas or the laboratory plasmas, we calculated for $T_{\mathrm{e}}<20 \mathrm{eV}$ and $10^{11}<n_{\mathrm{e}}<10^{14} \mathrm{~cm}^{-3}$.

In the application of the CR model, the ratio of the $p$ and $q$-states population, $n(p) / n(q)$, rather than that of the intensity was used. Visible transitions from $p$ or $q(n \geq 3)$ to the $n=2$ state (He I Balmer series) are suitable for the present purpose. Note that the states are sometimes represented by the transition wavelength in the air.

As can be seen in the calculated results of the population ratio $n(587) / n(447)$, where the numbers in the parentheses denote the wavelength in $\mathrm{nm}$, if $T_{\mathrm{e}}<2 \mathrm{eV}$, the ratio is applicable to the $T_{\mathrm{e}}$ measurement (A). If $2<T_{\mathrm{e}}<4 \mathrm{eV}$, the ratio is applicable for the $n_{\mathrm{e}}$ measurement for $10^{12}$ $10^{13} \mathrm{~cm}^{-3}(\mathrm{~B})$. In $10^{11}<n_{\mathrm{e}}<10^{12} \mathrm{~cm}^{-3}, T_{\mathrm{e}}$ is measurable for $5-8 \mathrm{eV}(\mathrm{C})$. In $10<T_{\mathrm{e}}<20 \mathrm{eV}, n_{\mathrm{e}}$ is measurable for $4 \times 10^{11}-2 \times 10^{12} \mathrm{~cm}^{-3}$. (D) Therefore, if one roughly anticipates the parameter in this region, one can measure these parameters only from this ratio. The ratio $n(492) / n(447)$ is applicable to the $T_{\mathrm{e}}$ measurement in a rather higher density regime, $10^{13}<n_{\mathrm{e}}<10^{14} \mathrm{~cm}^{-3}(\mathrm{E})$. Also, in $10<T_{\mathrm{e}}<20 \mathrm{eV}$, there is some dependence on $n_{\mathrm{e}}$ in $1 \times 10^{11}-2 \times 10^{12} \mathrm{~cm}^{-3}(\mathrm{~F})$, but the sensitivity may not be very good.

On the other hand, the resonant transition $2{ }^{1} \mathrm{~S}-3{ }^{1} \mathrm{P}$ $(501 \mathrm{~nm})$ is influenced by the radiation trapping so that this line can be used to check the obtained results including the neutral density.

We are applying this system to pure helium plasma in the MAP(material and plasma)-II steady-state linear divertor/edge plasma simulator at the University of Tokyo [13]. Preliminary results have revealed that the precise intensity calibration free from the ambiguous effect of the leakbands is crucial, since the value of the intensity ratio directly corresponds to the parameters. For example, the region (E) in Fig. 5 shows that about a $10 \%$ error in the intensity ratio leads to the $1 \mathrm{eV}$ difference in the $T_{\mathrm{e}}$. The accuracy of the measurement is expected to be improved by adopting the low-pass or pass-band filter to separate a tunable range into two domains.

\section{Summary and Future Prospects}

The imaging spectrometry of plasmas using a liquid crystal-based wavelength-tunable filter (Lyot filter) is proposed. With the help of a CR model, the image of the inten- sity ratio can be obtained, from which the parameter image can be determined. The usable lines in He I have been evaluated and the parameter range, where the intensity ratio between these lines can be applicable, was evaluated.

In the case of axi-symmetric plasma, the 3-D structure of the parameter can be deduced by performing an Abel inversion. Application to the divertor simulator MAP-II is underway.

In order to better determine the parameters, it may be useful to make use of the monochromator for the single viewing chord to cross-check the line ratio, or to compare with a direct measurement of $T_{\mathrm{e}}$ or $n_{\mathrm{e}}$ such as an electrical probe or Thomson scattering, to calibrate the conversion factor from the CCD counts to the photon flux. Furthermore, since the method yields a wide range of the plasma profile in high resolution, the accuracy of the Abel inversion to determine the local value from the line integrated data is quite high. This feature is useful for extrapolating the data obtained at the limited spatial points [14].

\section{Acknowledgment}

This work was supported in part by NIFS Collaborative Research Program (NIFS04KOAB009).

[1] T. Fujimoto, J. Quant. Spectrosc. Radiat. Transfer 21, 439 (1979).

[2] B. Lyot, "Optical Apparatus With Wide-Field Using Interference of Polarized Light," C.R. Acad. Sci. (Paris), 197, 1593 (1933).

[3] G.A. Kopp, M.J. Derks, D.F. Elmore et al., Appl. Opt. 36, 291-296 (1997).

[4] J. Staromlynska, S.M. Rees, and M.P. Gillyon, Appl. Opt. 37, 1081-1088 (1998).

[5] J. Bland-Hawthorn, W. Breugel, P.R. Gillingham et al., Astrophysical Journal 563, 611(2001).

[6] M. Goto, J. Quant. Spectrosc. Radiat. Transfer 76, 331 (2003).

[7] B. Schweer, G. Mank, A. Pospieszczyk et al., J. Nucl. Mater. 196-198, 174 (1992).

[8] S. Sasaki, S. Takamura, S. Watanabe et al., Rev. Sci. Instrum. 67, 3521 (1996).

[9] S. Kajita, N. Ohno S. Takamura and T. Nakano, Phys. Plasmas 13, 013301 (2006).

[10] M. Otsuka, R. Ikee and K. Ishii, J. Quant. Spectrosc. Radiat. Transfer, 21, 41 (1979).

[11] B. Xiao, S. Kado, S. Kajita and D. Yamasaki, Plasma Phys. Control. Fusion 46, 653 (2004).

[12] Y. Iida, S. Kado, A. Okamoto et al., J. Plasma Fusion Res. SERIES, 7, 123 (2006).

[13] S. Kado, Y. Iida, S. Kajita et al., J. Plasma Fusion Res. 81, 810 (2005).

[14] Y. Kuwahara, S. Kado, A. Okamoto et al., Plasma Fusion Res. 2, S1081 (2007). 